\title{
Diacronie
}

Studi di Storia Contemporanea

$\mathrm{N}^{\circ} 9,1 \mid 2012$

Quando la classe operaia andava in paradiso

\section{Nota introduttiva n. 9 - gennaio 2012}

\section{Luca Bufarale e Fausto Pietrancosta}

\section{(2) OpenEdition}

\section{Journals}

\section{Edizione digitale}

URL: http://journals.openedition.org/diacronie/2908

DOI: $10.4000 /$ diacronie.2908

ISSN: 2038-0925

\section{Editore}

Association culturelle Diacronie

\section{Notizia bibliografica digitale}

Luca Bufarale e Fausto Pietrancosta, « Nota introduttiva n. 9 - gennaio 2012 », Diacronie [Online], № 9 ,

1 | 2012, Messo online il 29 janvier 2012, consultato il 24 septembre 2020. URL : http://

journals.openedition.org/diacronie/2908; DOI : https://doi.org/10.4000/diacronie.2908

Questo documento è stato generato automaticamente il 24 settembre 2020.

Creative Commons License 


\title{
Nota introduttiva n. 9 - gennaio 2012
}

\author{
Luca Bufarale e Fausto Pietrancosta
}

«Tutti noi ce la prendiamo con la storia / ma io dico che la colpa è nostra / è evidente che la gente è poco seria / quando parla di sinistra o destra»: così iniziava un famoso pezzo di Giorgio Gaber di alcuni anni fa. La distinzione destra/ sinistra, pare suggerire il cantautore, è talmente entrata nel pensare comune da risultare quasi usurata: tutti ne parlano, ma nessuno sa bene in fondo di cosa si

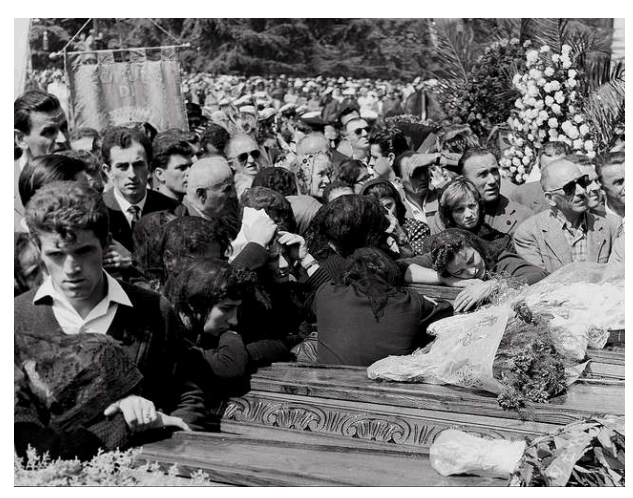
tratti, al punto da diventare quasi una questione di stereotipi, di mode passeggere («Fare il bagno nella vasca è di destra / far la doccia invece è di sinistra / un pacchetto di Marlboro è di destra / di contrabbando è di sinistra...»). Del resto, negli ultimi venti o trent'anni abbiamo spesso sentito ripetere, in Italia ma anche in altri paesi europei, illeit motiv "destra e sinistra non esistono più" oppure "non c'è più la sinistra di una volta". Secondo alcune indagini demoscopiche, persino in Francia - il paese in cui i termini sinistra/destra sono stati utilizzati, prima che altrove, nella definizione delle identità politiche - sembra che siano in molti a ritenere superata questa distinzione ${ }^{1}$. Al di là della polemica politica sull'attualità, ci si potrebbe chiedere se nell'analisi storica i due termini mantengano ancora una qualche funzionalità. $\mathrm{A}$ nostro avviso, la risposta è sì, a condizione che le due nozioni, come sottolinea giustamente Norberto Bobbio, non vengano intese in modo assoluto. Destra e Sinistra, infatti, «sono concetti relativi». «Non sono concetti sostantivi od ontologici. Non sono qualità intrinseche dell'universo politico. Sono luoghi dello "spazio politico". Rappresentano una determinata tipologia politica, che non ha niente a che vedere con l'ontologia politica: non si è di destra o di sinistra nello stesso senso per cui si dice che si è "comunisti", o "liberali", o "cattolici" $»^{2}$. In altri termini, destra e sinistra non sono 
parole che designano contenuti fissati una volta per sempre. Possono designare diversi contenuti secondo i tempi e le situazioni. Marco Revelli fa l'esempio dello spostamento della sinistra ottocentesca dal movimento liberale a quello democratico, sino a quello socialista. Ciò che è di sinistra è tale solo rispetto a ciò che è di destra. «Il fatto che destra e sinistra rappresentino una opposizione vuol dire semplicemente che non si può essere contemporaneamente di destra e di sinistra. Ma ciò non dice nulla sul contenuto delle due parti contrapposte. L'opposizione resta, anche se i contenuti dei due opposti possono cambiare» ${ }^{3}$.

2 Non si può però applicare la distinzione sinistra/destra in tutti i paesi e per tutte le epoche storiche. Affinché questa abbia un senso sono necessarie probabilmente alcune condizioni: la permanenza di un minimo di dibattito politico svolto in sedi pubbliche; il riconoscimento della cittadinanza a tutti gli individui; una nozione sufficientemente condivisa - ma discussa e non affermata autoritariamente - di progresso; il mantenimento della distinzione fra le categorie di uguaglianza e disuguaglianza; la costante tensione tra esigenze di emancipazione e difesa della tradizione, ma anche la presenza di un conflitto sociale che riesce a trovare canali legalitari per esprimersi e, non da ultimo, la sussistenza, all'interno stesso delle posizioni di destra e di sinistra, del binomio estremismo-moderazione. È innegabile che il concetto di sinistra nato proprio con la rivoluzione francese ${ }^{4}$, ovvero con l'esperienza che più ha costituito una svolta nella definizione di un'idea storica e laica di progresso, continua a nutrirsi ancora oggi di questi e di altri elementi costitutivi. Negli stati a regime autoritario o totalitario, dove tali condizioni non esistono, risulta a nostro avviso problematico utilizzare la distinzione sinistra/destra sia per connotare quei regimi, sia soprattutto per analizzarne le divisioni interne. In tal senso è fuorviante parlare, come spesso si fa, di "totalitarismi di destra" e "totalitarismi di sinistra" 5 . Stalin non può essere definito "di sinistra" più di quanto Hitler non possa essere definito "di destra" e ciò semplicemente perché questi due personaggi (e i regimi che li sostenevano) non ammettevano qualcuno "alla destra o alla sinistra di loro stessi". Anche definizioni come "fascismo di sinistra", per indicare quei settori del regime mussoliniano inclini ad usare la fraseologia socialisteggiante del fascismo sansepolcrista e a coltivare il mito della "terza via corporativista", appaiono quantomeno discutibili, così come l'utilizzo della contrapposizione destra/sinistra in contesti storici precedenti alla rivoluzione francese ${ }^{6}$.

3 In questa prospettiva il numero invernale di Diacronie affronta l'evoluzione delle sinistre europee nel trentennio 1945-1975. Annoverando contributi che spaziano a livello sia tematico che cronologico e che presentano talvolta un approccio comparativo, il numero tenta di fornire alcuni spunti su temi fondamentali con i quali i partiti e le generazioni politiche di sinistra si sono dovute confrontare. Questo periodo, definito nella nota opera sul XX secolo di Eric J. Hobsbawm come l'"età dell'oro" ${ }^{7}$ e dalla storiografia economica francese come les trente glorieuses ${ }^{8}$, ha rappresentato una fase della storia europea in cui alla ricostruzione post-bellica ha fatto seguito una decisa e costante crescita economica, inizialmente favorita dagli Stati Uniti, che si è accompagnata a politiche di redistribuzione della ricchezza e a mutamenti sociali e culturali indotti dall'avvento su larga scala dei fenomeni caratteristici della società di massa. Probabilmente, mai come in questo trentennio, almeno nei paesi dell'Europa occidentale, la contrapposizione destra-sinistra ha rappresentato l'elemento centrale dello scontro politico. Le sinistre d'ispirazione socialdemocratica si ridefiniscono sulla 
base di politiche fondate sull'universalità dei diritti sociali, come mette in evidenza l'articolo di Carlo Andrea Stazzi "“And Now - Win the peace!". I laburisti inglesi e il Welfare State (1945-1948)», oppure sul ruolo dinamico dei salari e sul pieno impiego, come nel caso delle socialdemocrazie scandinave analizzate da Paolo Borioni in «La socialdemocrazia nordica e la "sfida democratica al capitalismo"». I partiti comunisti italiano e francese, usciti rafforzati dalla Resistenza e decisi a portare avanti, almeno sino a quando la "guerra fredda" non glielo impedisce, una politica di collaborazione con i partiti "borghesi" e di ricostruzione dell'ordinamento democratico, ridefiniscono il concetto di nazione per legittimare il loro ruolo all'interno della storia dei rispettivi paesi: lo chiarisce bene, approfondendo analogie e differenze tra i due partiti, Martina Martignoni in «Due vie democratiche al socialismo? PCI, PCF e il concetto di nazione tra il 1944 e il 1947». L'apporto dei partiti di sinistra - nel caso specifico della socialdemocrazia tedesca - alla questione della denazificazione e della formazione di una coscienza antifascista è il tema del saggio di Jens Späth «Un antifascista e democratico particolare: il socialdemocratico bavarese Wilhelm Hoegner». L'influenza della "guerra fredda" e il ruolo dell'URSS nella demarcazione dei confini politici è uno degli aspetti del profilo di una figura emblematica di combattente antifascista come Sandro Pertini così come viene tracciato da Gianluca Scroccu in «Pacifismo, frontismo e autonomia: Pertini, il Psi e la "fase calda" della guerra fredda (1945-1950)». Il tentativo di superare le contrapposizioni - non solo di politica internazionale ma anche culturali - tra Est e Ovest emerge nella vicenda del gruppo di Unità Popolare raccontata da Roberto Colozza in «Guardare lontano. Modelli, esplorazioni e collaborazioni internazionali del movimento di Unità Popolare (1953-1957)». I cambiamenti indotti dal boom economico con le sue conseguenze sui fenomeni migratori inducono spesso $i$ partiti di massa a rivedere strategie e modelli organizzativi: è il caso del PCI dei primi anni sessanta affrontato da Michelangela Di Giacomo in «Pci e migrazioni interne nella Torino del "miracolo"».

4 Il rapporto tra i partiti di sinistra e il difficile processo di unificazione europea è il tema di fondo di vari articoli del numero. Brian Shaev in «The French Socialist and German Social Democratic Parties and the Future of the Working Class in the European Coal \& Steel Community, 1948-1954» mette in evidenza le differenze tra i socialisti francesi, maggiormente fiduciosi sulle possibilità espansive offerte dalla Comunità europea del carbone e dell'acciaio e dal Piano Schuman, e i socialdemocratici tedeschi, più timorosi delle ricadute occupazionali del piano per l'industria della Germania Ovest. Enrico Pugliese nel saggio «Nazionale e globale nella rinascita dell'Internazionale socialista (1945-1951)» analizza come le socialdemocrazie dei vari paesi attuano un nuovo tipo di collaborazione centrato sulla gestione della governance europea intesa però più come ridefinizione nel rapporto tra stati che come sforzo di creazione di un'unità federale tra i vari paesi. L'adesione del Partito socialista italiano prima e di quello comunista poi al progetto europeo viene delineata da Maria Serena Adesso in «Il consenso delle sinistre italiane all'integrazione europea (1950-1969)».

5 Se i partiti socialdemocratici individuano gradualmente nel processo di unificazione europea una delle modalità d'intervento per portare avanti le loro istanze di maggiore eguaglianza e di regolazione del capitalismo, trovando in ciò, almeno a partire dalla fine degli anni sessanta, un terreno comune con il Partito comunista italiano, le sinistre rivoluzionarie fanno da subito della lotta anticolonialista e antimperialista uno dei loro punti di forza. Le iniziative a favore del movimento di liberazione algerino contro la colonizzazione francese sono l'occasione per stimolare nuovi incontri tra militanti di 
gruppi spesso con tradizioni e storie molto diverse tra loro - anarchici, trotzkisti, socialisti di sinistra ecc. - come mostrano i saggi di Nedjib Sidi-Moussa («Face à la guerre d'Algérie: transactions anticoloniales et reconfigurations dans la gauche française») e di Mathieu Le Tallec («L'unité d'action des trotskystes, anarchistes et socialistes de gauche autour de l'anticolonialisme et de l'anti-bonapartisme [1954-1958]»). L'anarchismo italiano nelle sue contaminazioni con altri movimenti libertari in una fase relativamente poco conosciuta della sua storia è l'argomento di Giorgio Sacchetti in «Eretici e libertari. Il movimento anarchico in Italia 1945-1973». I difficili rapporti tra due formazioni politiche di provenienza differente, ma accomunate dal tentativo di interpretare sino in fondo le istanze di rinnovamento emerse nel biennio 1968-1969 ed entrate per questo in conflitto con i loro partiti di origine, vengono discussi nell'articolo di Antonio Lenzi «La resistibile ascesa verso l'unificazione. L'incontro tra il Pdup e "il manifesto"». L'approccio delle organizzazioni della "nuova sinistra" italiana emersa con il Sessantotto rispetto alle lotte condotte dai movimenti "nazionalitari" in Europa - ad esempio nei paesi baschi o nell'Irlanda del Nord - e più in generale il rapporto tra i partiti che si richiamano agli ideali socialisti $\mathrm{e}$ internazionalisti e la questione nazionale costituiscono i temi del saggio di Paolo Perri «Nazionalità e lotta di classe. La Nuova Sinistra e i nazionalismi periferici».

Chiudono il numero le interviste a due storici che si sono occupati delle vicende politiche italiane ed europee e che conoscono bene la storia dei partiti di sinistra, in qualche caso anche per avervi militato. È nostro auspicio che esse possano, insieme agli articoli, fornire alcune suggestioni e nuovi spunti per il dibattito, oggi più che mai attuale, sul futuro delle sinistre partendo da quella che è stata la loro storia.

\section{NOTE}

1. REVELLI, Marco, Sinistra destra. L'identità smarrita, Roma-Bari, Laterza, 2007, pag. V.

2. BOBBIO, Norberto, Destra e Sinistra. Ragioni e significati di una distinzione politica, Roma, Donzelli Editore, 2004.

3. REVELLI, Marco, «Sinistra/Destra. Conversazione sull'attualità di un'antitesi», in Quaderni del Cric, 1, marzo-giugno 1986.

4. È noto come uno degli eventi alla base della distinzione destra/sinistra fu la seduta dell'Assemblea Costituente a Versailles il 28 agosto 1789, quando, per facilitare il conteggio dei voti, i sostenitori del veto regio si sedettero alla destra del presidente dell'assemblea e gli oppositori a sinistra. Questa distinzione si ripropose in seguito, consolidando il binomio sinistrarivoluzione e quello destra-reazione, e si diffuse gradualmente anche in altri paesi. Almeno durante l'Ottocento, tuttavia, essa rimase confinata perlopiù nell'ambito della vita parlamentare, estendendosi al dibattito politico generale soltanto dall'inizio del Novecento. Cfr. GAUCHET, Marcel, Storia di una dicotomia: la destra e la sinistra, Milano, Anabasi, 1994; CRAPEZ, Marc, «De quand date le clivage gauche/droite en France?», in Revue française de science politique, 1/1998, pp. 42-75.

5. Sul concetto di totalitarismo cfr. ARENDT, Hannah, Le origini del totalitarismo, Milano, Edizioni

di Comunità, 1967. Per un raffronto tra differenti regimi totalitari sulla base della 
contrapposizione destra/sinistra vedi PELLICANI, Luciano, Lenin e Hitler. I due volti del totalitarismo, Soveria Mannelli, Rubbettino, 2008. A differenza della Arendt, che parla di totalitarismo al singolare e che vi include oltre al nazismo soltanto il regime stalinista e non l'URSS dei primi tempi o quella post-1956 di Chruščëv, Pellicani parla di totalitarismi al plurale (sia "di destra" che "di sinistra") e vi include il regime bolscevico sin dai suoi inizi.

6. Il concetto di fascismo di sinistra, inizialmente usato soprattutto dalla pubblicistica vicina a certi settori del Movimento Sociale Italiano, si è poi diffuso anche in ambito storiografico (cfr. ad esempio PARLATO, Giuseppe, La sinistra fascista. Storia di un progetto mancato, Bologna, Il Mulino, 2000).

7. HOBSBAWM, Eric J., Il secolo breve 1914-1991, Milano, Rizzoli, 1997.

8. FOURASTIÉ, Jean, Les trente glorieuses ou la révolution invisible de 1946 à 1975, Paris, Fayard, 1979; CHEVALLIER, François-Xavier, Le Bonheur economique: les trente glorieuses sont devant nous, Paris, Albin Michel, 1998.

\section{AUTORI}

\section{LUCA BUFARALE}

Luca Bufarale ha conseguito nel 2008 la laurea specialistica in storia d'Europa presso l'Università di Bologna e nel 2012 il dottorato di ricerca in scienze storiche (indirizzo contemporaneo) presso l'Università di Padova con una tesi dal titolo La giovinezza politica di Riccardo Lombardi (1919-1949). Ha condotto i suoi primi studi sull'Unione Sovietica pubblicando il saggio «Per un socialismo di mercato. Aspetti del dibattito economico in URSS negli anni sessanta» (in Storicamente, 2, 2006, http://www.storicamente.org/05_studi_ricerche/02bufarale.htm); successivamente ha indirizzato le sue ricerche sulla figura politica e intellettuale del leader azionista e poi socialista Riccardo Lombardi. È membro della Società italiana per lo studio della storia contemporanea e dall'Association for the study of Modern Italy. Su Lombardi ha pubblicato articoli su «Il Ponte», sugli «Annali della Fondazione Ugo La Malfa» e su «Diacronie. Studi di Storia Contemporanea». Ha partecipato come relatore ad un convegno promosso dall'Associazione nazionale Riccardo Lombardi (Torino, 7 novembre 2009) e alla sesta edizione di "Giellismo e azionismo: cantieri aperti" (Torino, 6 - 8 maggio 2010). Su Lombardi ha pubblicato un articolo su «Il Ponte» (marzo 2010). È di prossima pubblicazione un suo saggio negli «Annali della Fondazione Ugo La Malfa».

URL: < http://www.studistorici.com/2010/12/02/luca-bufarale/ >

\section{FAUSTO PIETRANCOSTA}

Fausto Pietrancosta ha conseguito il titolo di dottore di ricerca in Storia presso l'Università di Bologna con una tesi inerente le relazioni tra istituzioni politiche e intervento pubblico in economia nella prospettiva del coordinamento tra amministrazioni centrali ed ente regionale siciliano. Già dottore magistrale in Storia d'Europa, presso la stessa Università con una tesi in Storia dello Stato italiano sul coordinamento costituzionale e l'avvio dell'autonomia regionale siciliana, ha svolto attività di ricerca presso l'Archivio Storico e la biblioteca dell'Istituto Luigi Sturzo di Roma, presso l'Assemblea regionale siciliana, proseguendo poi l'attività di ricerca presso gli archivi degli enti pubblici economici in Sicilia e presso la biblioteca SVIMEZ di Roma. I 
suoi interessi sono rivolti allo studio dell'evoluzione storica delle autonomie regionali nell'Italia del secondo dopoguerra e delle politiche di intervento a favore dello sviluppo industriale nel Mezzogiorno.

URL: < http://www.studistorici.com/2008/09/14/fausto-pietrancosta/ > 